\title{
AUTHOR INDEX Volume 18
}

\begin{abstract}
Achdou, Y., Camilli, F. \& Capuzzo Dolcetta, I., Homogenization of Hamilton-Jacobi equations: Numerical methods

Adams, J., Conti, S., Desimone, A. \& Dolzmann, G., Relaxation of some transversally isotropic energies and applications to smectic A elastomers
\end{abstract}

Albeverio, S. \& Alt, W., Stochastic dynamics of viscoelastic skeins: Condensation waves and continuum limits

Alt, W., see Albeverio

Andreu, F., Igbida, N., Mazón, J. M. \& Toledo, J., Obstacle problems for degenerate elliptic equations with nonhomogeneous nonlinear boundary conditions

Aurelle, D., see Jabin

Auricchio, F., Mielke, A. \& Stefanelli, U., A rate-independent model for the isothermal quasi-static evolution of shape-memory materials

Barrett, J. W. \& Süli, E., Existence of global weak solutions to dumbbell models for dilute polymers with microscopic cut-off

Beirão da Veiga, L. \& Lovadina, C., An interpolation theory approach to shell eigenvalue problems

Bellomo, N. \& Brezzi, F., Traffic, crowds, and swarms

Bellomo, N. \& Dogbé, C. On the modelling crowd

18 (2008) 1115

$18(2008) 1$

18S (2008) 1149

18S (2008) 1149

18 (2008) 1869

18 (2008) 1927

18 (2008) 125

18 (2008) 935

18 (2008) 2003 dynamics: From scaling to hyperbolic macroscopic models

Bellomo, N., Li, N. K. \& Maini, P. K., On the foundations of cancer modelling: Selected topics, speculations, and perspectives

Bernardi, C., Girault, V. \& Rajagopal, K. R., Discretization of an unsteady flow through a porous solid modeled by Darcy's equations

Berthelin, F., Degond, P., Le Blanc, V., Moutari, S., Rascle, M. \& Royer, J., A traffic-flow model with constraints for the modeling of traffic jams

Berthon, C. \& Coquel, F., Shock layers for turbulence models

Bertoluzza, S., Falletta, S. \& Manzini, G., Efficient design of residualbased stabilization techniques for the three fields domain decomposition method

Bertotti, M. L. \& Delitala, M., On the existence of limit cycles in opinion formation processes under time periodic influence of persuaders

Bertozzi, A. L., see Short
18S (2008) 1145

18S (2008) 1317

$18(2008) 593$

18 (2008) 2087

18S (2008) 1269

18 (2008) 1443

18 (2008) 973

18 (2008) 913

18S (2008) 1249 
Boccardo, L., Orsina, L. \& Porretta, A., Existence of finite energy solutions for elliptic systems with $L^{1}$-valued nonlinearities

Bonnet-Ben Dhia, A.-S., Ciarlet, P., Jr., \& Zwölf, C. M., A new compactness result for electromagnetic waves. Application to the transmission problem between dielectrics and metamaterials

Bouchitté, G. Gangbo, W. \& Seppecher, P., Michell trusses and lines of principal action

Brantingham, P. J., see Short

Brezzi, F., see Bellomo

Brunk, M. \& Jüngel, A., Simulation of thermal effects in optoelectronic devices using coupled energy-transport and circuit models

Bürger, R., García, A. \& Kunik, M., A generalized kinetic model of sedimentation of polydisperse suspensions with a continuous particle size distribution

Cagnetti, F., A vanishing viscosity approach to fracture growth in a cohesive zone model with prescribed crack path

Camilli, F., see Achdou

Canavesio, C., see Coscia

Capuzzo Dolcetta, I., see Achdou

Castillo, E., Luceño, A., \& Pedregal, P., Composition functionals in calculus of variations. Application to products and quotients

Castro, C., Palacios, F. \& Zuazua, E., An alternating descent method for the optimal control of the inviscid Burgers equation in the presence of shocks

Chayes, L. B., see Short

Cheng, J., Choulli, M. \& Lin, J., Stable determination of a boundary

coefficient in an elliptic equation

Choulli, M., see Cheng

Christiansen, S. H., A

18 (2008) 669

18 (2008) 1605

$18(2008) 1571$

18S (2008) 1249

18S (2008) 1145

18 (2008) 2125

18 (2008) 1741

18 (2008) 1027

18 (2008) 1115

18S (2008) 1217

18 (2008) 1115

18 (2008) 47

18 (2008) 369

18S (2008) 1249 construction of spaces of compatible differential forms on cellular complexes

Ciarlet, P., Jr., see BonnetBen Dhia

Ciarlet, P. G., Gratie, L., Mardare, C. \& Shen, M., Saint Venant compatibility equations on a surface application to intrinsic shell theory

Conti, M., Gatti, S. \& Pata, V., Uniform decay properties of linear Volterra integrodifferential equations

Conti, S., see Adams

Coquel, F., see Berthon

Coscia, V. \& Canavesio, C., First-order macroscopic modelling of human crowd dynamics da Veiga, L. B., Hakula, H. \& Pitkäranta, J., Asymptotic and numerical analysis of the eigenvalue problem for a clamped cylindrical shell

D'Angelo, C. \& Quarteroni, A., On the coupling of $1 \mathrm{D}$ and $3 \mathrm{D}$ diffusion-reaction equations. Application to tissue perfusion problems

D'Apice, C. \& Piccoli, B., Vertex flow models for vehicular traffic on networks

D’Orsogna, M. R., see Short

Degond, P. \& Motsch, S., Continuum limit of selfdriven particles with orientation interaction

Degond, P., see Berthelin

Delitala, M., see Bertotti

Desimone, A., see Adams

Dogbé, C., see Bellomo

Dogbé, C., Fluid dynamic limits for gas mixture I. Formal derivations

Dolzmann, G., see Adams

Donato, M. B., Milasi, M. \& Vitanza, C., Quasivariational approach
18 (2008) 107

18 (2008) 107

18 (2008) 739

18 (2008) 1605

18 (2008) 165

$18(2008) 21$

18 (2008) 1

18 (2008) 1443

18S (2008) 1217

18 (2008) 1983

18 (2008) 1481

18S (2008) 1299

18S (2008) 1249

18S (2008) 1193

18S (2008) 1269

18 (2008) 913

18 (2008) 1

18S (2008) 1317

18 (2008) 1633

18 (2008) 1 
of a competitive economic equilibrium problem with utility function: Existence of equilibrium

Drago, C. R. \& Pinnau, R., Optimal dopant profiling based on energytransport semiconductor models

Duan, R.-J., Li, M.-R. \& Yang, T., Propagation of singularities in the solutions to the Boltzmann equation near equilibrium

Ducrot, A. \& Langlais, M., Travelling waves in invasion processes with pathogens

Falletta, S., see Bertoluzza

Fang, N. \& Ying, L.-A., Three-dimensional exterior problem of Darwin model and its numerical computation

Farina, A., Fasano, A. \& Mikelić, A., On the equations governing the flow of mechanically incompressible, but thermally expansible, viscous fluids

Fasano, A., see Farina

Feireisl, E., Novotný, A. \& Petzeltová, H., On the incompressible limit for the NavierStokes-Fourier system in domains with wavy bottoms

Frank, M., Herty, M. \& Schäfer, M., Optimal treatment planning in radiotherapy based on Boltzmann transport calculations

Friedman, A. \& $\mathrm{Hu}$, B., The role of oxygen in tissue maintenance: Mathematical modeling and qualitative analysis

Gangbo, W., see Bouchitté

Gao, W. \& Zhu, C., Asymptotic decay toward the planar rarefaction waves for a model system of the radiating gas in two dimensions
García, A., see Bürger

$18(2008) 195$

18 (2008) 1093

18 (2008) 325

18 (2008) 973

18 (2008) 1673

18 (2008) 813

18 (2008) 813

18 (2008) 291

$18(2008) 573$

18 (2008) 1409

18 (2008) 1571

18 (2008) 511

$\mathrm{Hu}$, B., see Friedman
Garcke, H., Nestler, B., Stinner, B. \& Wendler, F., Allen-Cahn systems with volume constraints

Gatti, S., see Conti

Girault, V., see Bernardi

Gobbi, R., Palpacelli, S. \& Spigler, R., Numerical treatment of a nonlinear nonlocal transport equation modeling crystal precipitation

Götz, T., Klar, A., Unterreiter, A. \& Wegener,

18 (2008) 1741

18 (2008) 1347

18 (2008) 21

18 (2008) 2087

18 (2008) 1505

18 (2008) 1829

18 (2008) 165

18 (2008) 215 fluid-elastic interaction model

Gwiazda, P. \&

Świerczewska-Gwiazda,

A., On non-Newtonian fluids with a property of rapid thickening under different stimulus

18 (2008) 1073

Haddar, H., Joly, P. \& Nguyen, H.-M., Generalized impedance boundary conditions for scattering problems from strongly absorbing obstacles: The case of Maxwell's equations

Hakula, H., see da Veiga

Haramoto, K., see Ide

Hermann, M. \& Timokha, A., Modal modelling of the nonlinear resonant fluid sloshing in a rectangular tank II: Secondary resonance

Herty, M., see Frank

Ide, K., Haramoto, K. \& Kawashima, S., Decay property of regularityloss type for dissipative Timoshenko system

18 (2008) 1787

18 (2008) 1983

18 (2008) 647

18 (2008) 1845

18 (2008) 573

18 (2008) 1409

Ide, K. \& Kawashima, S., Decay property of regularity-loss type and nonlinear effects for dissipative Timoshenko system 
Igbida, N., see Andreu

Jabin, P.-E., Lemesle, V. \& Aurelle, D., A continuous size-structured red coral growth model

Joly, P., see Haddar

Jüngel, A., see Brunk

Kawashima, S., see Ide

Kawashima, S., see Ide

Kazmierczak, B. \& Volpert, V., Travelling calcium waves in systems with non-diffusing buffers

Klar, A., see Götz

Knees, D., Mielke, A. \& Zanini, C., On the inviscid limit of a model for crack propagation

Kohr, M., Raja Sekhar, G. P. \& Wendland, W. L., Boundary integral equations for a threedimensional StokesBrinkman cell model

Kunik, M., see Bürger

Langlais, M., see Ducrot

Le Blanc, V., see Berthelin

Lemesle, V., see Jabin

Li, H.-L., Zhang, G. \& Zhang, K., Algebraic time decay for the bipolar quantum hydrodynamic model

Li, M.-R., see Duan

Li, N. K., see Bellomo

Lin, J., see Cheng

Liu, H. \& Sparber, C., Rigorous derivation of the hydrodynamical equations for rotating superfluids

Lovadina, C., see Beirão da Veiga

Luceño, A., see Castillo

Maini, P. K., see Bellomo

Manzini, G., see Bertoluzza

Mardare, C., see Ciarlet

Markowich, P. A., see Wu

Mazón, J. M., see Andreu

Mielke, A., see Auricchio

Mielke, A., see Knees

Migórski, S., Ochal, A. \& Sofonea, M., Integrodifferential hemivariational inequalities with applications to viscoelastic frictional contact

Mikelić, A., see Farina

Milasi, M., see Donato
18 (2008) 1869

18 (2008) 1927

18 (2008) 1787

18 (2008) 2125

18 (2008) 647

18 (2008) 1001

18 (2008) 883

18 (2008) 1829

18 (2008) 1529

18 (2008) 2055

18 (2008) 1741

18 (2008) 325

18S (2008) 1269

18 (2008) 1927

18 (2008) 859

18 (2008) 1093

18 (2008) 593

18 (2008) 107

18 (2008) 689

18 (2008) 2003

18 (2008) 47

18 (2008) 593

18 (2008) 973

18 (2008) 165

18 (2008) 443

18 (2008) 1869

18 (2008) 125

18 (2008) 1529

18 (2008) 271

$18(2008) 813$

18 (2008) 351
Morin, P., Siebert, K. G. \& Veeser, A., A basic convergence result for conforming adaptive finite elements

Motsch, S., see Degond

Moutari, S., see Berthelin

Munnier, A., On the selfdisplacement of deformable bodies in a potential fluid flow

Negri, M. \& Ortner, C., Quasi-static crack propagation by Griffith's criterion

Nestler, B., see Garcke

Nguyen, H.-M., see Haddar

Novotný, A., see Feireisl

Oberman, A. M., Computing the convex envelope using a nonlinear partial differential equation

Ochal, A., see Migórski

Orsina, L., see Boccardo

Ortner, C., see Negri

Padula, M., see Guidorzi

Palacios, F., see Castro

Palpacelli, S., see Gobbi

Pasour, V. B., see Short

Pata, V., see Conti

Pedregal, P., see Castillo

Petzeltová, H., see Feireisl

Piccoli, B., see D'Apice

Pinnau, R., see Drago

Pitkäranta, J., see da Veiga

Plotnikov, P. I., see Guidorzi

Porretta, A., see Boccardo Qin, Y. M. \& Zhao, Y. L., Global existence and asymptotic behavior of the compressible Navier-Stokes equations for a $1 \mathrm{D}$ isothermal viscous gas

Quarteroni, A., see D'Angelo

Rajagopal, K. R., see Bernardi

Raja Sekhar, G. P., see Kohr

Ramdani, K. \& Shipman, S., Transmission through a thick periodic slab

Rascle, M., see Berthelin

Rohde, C. \& Yong, W.-A., Dissipative entropy and global smooth solutions
18 (2008) 707

18S (2008) 1193

18S (2008) 1269

18 (2008) 1945

18 (2008) 1895

18 (2008) 1347

18 (2008) 1787

18 (2008) 291

18 (2008) 759

18 (2008) 271

18 (2008) 669

18 (2008) 1895

18 (2008) 215

18 (2008) 369

18 (2008) 1505

18S (2008) 1249

18 (2008) 21

18 (2008) 47

18 (2008) 291

18S (2008) 1299

18 (2008) 195

18 (2008) 1983

18 (2008) 215

18 (2008) 669

18 (2008) 1383

18 (2008) 1481

18 (2008) 2087

18 (2008) 2055

18 (2008) 543

18S (2008) 1269 
in radiation hydrodynamics and magnetohydrodynamics

Royer, J., see Berthelin

Rozanova-Pierrat, A., Qualitative analysis of the Khokhlov-Zabolotskaya-Kuznetsov (KZK) equation

Scardia, L., Damage as $\Gamma$-limit of microfractures in anti-plane linearized elasticity

Schäfer, M., see Frank

Seppecher, P., see Bouchitté

Shen, M., see Ciarlet

Shipman, S., see Ramdani

Short, M. B., D'Orsogna, M. R., Pasour, V. B., Tita, G. E., Brantingham, P. J., Bertozzi, A. L. \& Chayes, L. B., A statistical model of criminal behavior

Siebert, K. G., see Morin

Sofonea, M., see Migórski

Sparber, C., see Liu

Spigler, R., see Gobbi

Stefanelli, U., see Auricchio

Stinner, B., see Garcke

Süli, E., see Barrett

Świerczewska-Gwiazda, A., see Gwiazda

Timokha, A., see Hermann

Tita, G. E., see Short

Toledo, J., see Andreu

Unterreiter, A., see Götz

Vauchelet, N., Diffusive transport of partially quantized particles: $L \log L$ solutions
18 (2008) 2151

18S (2008) 1269

18 (2008) 781

18 (2008) 1703

18 (2008) 573

18 (2008) 1571

18 (2008) 165

18 (2008) 543

18S (2008) 1249

18 (2008) 707

18 (2008) 271

18 (2008) 689

18 (2008) 1505

18 (2008) 125

18 (2008) 1347

18 (2008) 935

18 (2008) 1073

18 (2008) 1845

18S (2008) 1249

18 (2008) 1869

18 (2008) 1829

18 (2008) 489
Vazquez, J. L. \& Vitillaro, E., Wave equation with second-order nonstandard dynamical boundary conditions

Veeser, A., see Morin

Vitanza, C., see Donato

Vitillaro, E., see Vazquez

Volpert, V., see Kazmierczak

Wegener, R., see Götz

Wendland, W. L., see Kohr

Wendler, F., see Garcke

Wu, H., Markowich, P. A. \& Zheng, S., Global existence and asymptotic behavior for a semiconductor drift-diffusionPoisson model

$\mathrm{Xu}$, J. \& Zhu, Y., Uniform convergent multigrid methods for elliptic problems with strongly discontinuous coefficients

Yang, T., see Duan

Ying, L.-A., see Fang

Yong, W.-A., see Rohde

Zanini, C., see Knees

Zhang, G., see $\mathrm{Li}$

Zhang, K., see $\mathrm{Li}$

Zhang, S., An asymptotic analysis on the form of Naghdi type arch model Zhao, Y. L., see Qin

Zheng, S., see $\mathrm{Wu}$

Zhu, C., see Gao

Zhu, Y., see Xu

Zuazua, E., see Castro

Zwölf, C. M., see BonnetBen Dhia
18 (2008) 2019

18 (2008) 707

18 (2008) 351

18 (2008) 2019

18 (2008) 883

18 (2008) 1829

18 (2008) 2055

18 (2008) 1347

18 (2008) 443

18 (2008) 77

18 (2008) 1093

18 (2008) 1673

18 (2008) 2151

18 (2008) 1529

18 (2008) 859

18 (2008) 859

18 (2008) 417

18 (2008) 1383

18 (2008) 443

18 (2008) 511

18 (2008) 77

18 (2008) 369

18 (2008) 1605 